\title{
Transient Accelerating Scalar Models with Exponential Potential
}

\author{
W. P. Cui, Y. Zhang, Z. W. Fu \\ Key Laboratory for Researches in Galaxies and Cosmology CAS, \\ Department of Astronomy \\ University of Science and Technology of China \\ Hefei, Anhui, 230026, China
}

\begin{abstract}
We study a known class of scalar dark energy models in which the potential is of exponential type and the current accelerating era is transient. We find that, although a decelerating era will return in future, however, when extrapolating the model back to the earlier stages $(z \gtrsim 4)$, the scalar dark energy becomes dominant over the matter. So these models do not have the desired tracking behavior, and the predicted transient period of acceleration can not be adopted into the standard scenario of Big Bang cosmology. When couplings between the scalar field and the matter are introduced, the models still has the problem, only the time of deceleration return will be varied. To achieve re-deceleration, one has to turn to alternative models that are consistent with the standard Big Bang scenario.
\end{abstract}

\section{Introduction}

Cosmological observations have indicated that the universe is now in an accelerating expansion [1, 2, 3, 4, 5, 6]. Within the framework of general relativity, 
the cause of acceleration can be attributed to the existence of some dark energy, which makes up $\sim 70 \%$ of the total cosmic energy in the Universe. There are a number of possible candidates as the dark energy driving the accelerating expansion. The simplest one is the cosmological constant $\Lambda$, which, however, has the fine-tuning difficulty and the coincidence problem [7]. To overcome these difficulties within the framework of the theory of general relativity, dynamic dark energy models have been proposed, among them, one type is based on some scalar field, such as quintessence [8], phantom [9], k-essence [10], Tachyon field [11], quintom [12], Chaplygin gas [13], and other type is dynamic vector models based upon the Yang-Mills fields [14, 15, 16].

So far, there is no observational evidences whether the current cosmic acceleration is eternal or transient. Recently, the analysis of combined data of SN $\mathrm{la}+\mathrm{BAO}+\mathrm{CMB}$ made in Ref. [17] seems to indicate that the acceleration is slowing down. Most of dark energy models predict a scenarios that the acceleration will be eternal. As is known, in such an eternally accelerating universe, there is an event horizon, and causality exists in a limited spacetime. From the perspective of string theory, an asymptotically large space at infinity is required for the existence of conventional S-matrix, whose elements are connected with physical observables. Therefore, S-matrix is ill-defined in an eternal accelerating universe [18]. If one wants to save string theory as a theory of physics, one has to either look for alternatives to the conventional S-matrix, or to construct cosmological models, in which the current acceleration is a transient one and the decelerating expansion will eventually return.

Several dynamic dark energy models have been proposed to achieve the possibility of cosmic expansion that is accelerating currently, then will be decelerating in the future [19, 20, 21, 22, 23]. One model was explored in the context of $(4+n)$-dimensional gravity compactified on an $n$-dimensional time-varying compact manifold [19]. A class of braneworld models were also shown to admit a transient acceleration in certain regions of model parameters [20]. The transient acceleration was also examined in a scalar cosmological model in $d$-dimensions with exponential potentials, and a general solution was obtained flat RobertsonWalker metric [21]. An extension was made to the case with a generalized exponential potential of scalar field [22, 23]. Scalar fields with exponential potentials can occur generically in certain theories of particle physics, such as Kaluza-Klein theory with extra dimensions compactified [24], supersymmetry theories [25], and higher order gravity [26], and have been extensively studied in more general context for dark energy or for inflation as well [27, 28].

It is interesting to explore the possibility of transient acceleration in these 
general cosmological models. In particular, we want to check if these models can be extended to earlier epochs and consistent with the standard Big Bang scenario as a realistic dark energy model should be. So far in these scalar models of transient acceleration, either the cosmic matter component was not taken into consideration, or the dark energy was assumed to be independent of the matter. In the time-dependent $\Lambda(t)$ model [29] and the fluid dark energy model [30], interactions are introduced between dark energy and matter. Although so far there has not been any observational indication of a coupling between the dark energy and the matter, the dark energy as a dynamic scalar field could have interactions with other components, such as the matter. We will also study the models with coupling between scalar dark energy and matter, and to examine the impacts of the coupling upon the dynamic evolution behavior.

\section{The coupling model}

We consider a spatially flat $(k=0)$ Robertson-Walker spacetime with a metric $d s^{2}=d t^{2}-a^{2}(t) d \mathbf{x}^{2}$. The lagrangian of the scalar field that drives the acceleration is given by $\mathcal{L}=\frac{1}{2} \partial^{\mu} \phi \partial_{\mu} \phi-V(\phi)$. The energy density and pressure are $\rho_{\phi}=\frac{1}{2} \dot{\phi}^{2}+V(\phi), p_{\phi}=\frac{1}{2} \dot{\phi}^{2}-V(\phi)$, and the equation of state $w=p_{\phi} / \rho_{\phi}$. The dynamic expansion of universe is determined by the Friedmann equations:

$$
\begin{gathered}
H^{2}=\frac{8 \pi G}{3}\left(\rho_{\phi}+\rho_{m}+\rho_{r}\right), \\
\frac{\ddot{a}}{a}=-\frac{4 \pi G}{3}\left(\rho_{\phi}+3 p_{\phi}+\rho_{m}+\rho_{r}+3 p_{r}\right),
\end{gathered}
$$

where $H=\dot{a} / a$ is the expansion rate and its present value $H_{0}$ is the Hubble constant, $\rho_{m}$ and $\rho_{r}$ are the energy density of matter and radiation, respectively. The equations of evolution of the three components are given by

$$
\begin{gathered}
\dot{\rho}_{\phi}+3 H\left(\rho_{\phi}+p_{\phi}\right)=Q, \\
\dot{\rho}_{m}+3 H \rho_{m}=-Q, \\
\dot{\rho}_{r}+4 H \rho_{r}=0,
\end{gathered}
$$

where the coupling $Q$ is a generic coupling term and has a meaning of the energy exchange rate between the dark energy and matter. The non-coupling model is $Q=0$. When $Q>0$, the matter transfers energy into the dark energy. When $Q<0$, the dark energy transfers energy into the matter. We do not include the 
coupling between the dark energy and the radiation, which will not affect the following conclusion. Eq.(2) can be derived from from the set of equations (1), (3), (4) and (5). It has been shown that, in absence of the matter component in the non-coupling model [21, 22], for the current accelerating stage of expansion to be transient, i.e., for the expansion to turn into decelerating again, the scalar field potential $V(\phi)$ can take on the following form:

$$
\left.V(\phi)=\rho_{\phi 0}\left[1-\frac{\lambda}{6}(1+\alpha \sqrt{\sigma} \phi)^{2}\right)\right] \exp \left[-\lambda \sqrt{\sigma}\left(\phi+\frac{\alpha \sqrt{\sigma}}{2} \phi^{2}\right)\right]
$$

where $\rho_{\phi 0}$ is a constant energy density, $\sigma=8 \pi G / \lambda$, and $\alpha$ and $\lambda$ are two dimensionless, positive parameters of the model. In the context of this paper, they can take values around $\alpha, \lambda \sim 1$. In the limit $\alpha \rightarrow 0$ the potential in Eq.(6) reduces to an exponential potential, $V(\phi)=V_{0} \exp [-\sqrt{8 \pi G \lambda} \phi]$, a case that was examined in Ref.[21].

For convenience of computation, it is simpler to rewrite the set of equations, Eqs.(1), (3), (4)), in the following form,

$$
\begin{aligned}
h^{2} & =\frac{U(y)+x}{1-\frac{1}{2}\left(\frac{d y}{d N}\right)^{2}}, \\
\frac{d^{2} y}{d N^{2}}-\frac{3}{2}\left(\frac{d y}{d N}\right)^{3}+3 \frac{d y}{d N} & =\left(\frac{\Gamma}{\frac{d y}{d N}}+1.5 \frac{d y}{d N} x-U^{\prime}(y)\right) h^{-2}, \\
\frac{d x}{d N} & =-\Gamma-3 x,
\end{aligned}
$$

where $h \equiv H / H_{0}, N \equiv \ln a(t)=-\ln (1+z), y \equiv \sqrt{\frac{8 \pi G}{3}} \phi, x \equiv \rho_{m} / \rho_{c}$, $\Gamma \equiv Q / H \rho_{c}$, and $U(y) \equiv V(\phi) / \rho_{c}$. All of these quantities are dimensionless. Specifically, we take the model with $\Omega_{\phi}=0.73$ and $\Omega_{m}=0.27$ at $z=0$. The corresponding initial condition at $z=0$ is $x_{i}=0.27$, and $\phi_{i}=0$ and $\dot{\phi}_{i}=\sqrt{\lambda \rho_{\phi 0} / 3}$. The dynamical equations is then solved in the presence of matter component and radiation, yielding a transient acceleration about $z=0$ followed by the deceleration for the following three typical cases. Note that the parameter range of $\lambda$ and $\alpha$ for a transient acceleration in our model differ from those in Refs. [21, 22], which assumed the absence of matter.

For the non-coupling case $\Gamma=0$, Fig 1 shows the evolution of energy densities, $\rho_{\phi}(t), \rho_{m}(t)$, and $\rho_{r}(t)$, and Fig.2 shows the evolution of the deceleration parameter $q(z)=-\ddot{a} / a \dot{a}^{2}$ for various values of the parameter $\alpha$, larger values 
of $\alpha>0$ yield a shorter period of transient acceleration and an earlier return of deceleration. The special case with $\alpha=0$ corresponds to the the exponential potential [21], in which the current acceleration is eternal and deceleration will not return.

Fig. 3 show $\rho(t)$, and Fig. 4 show $q(z)$ for the coupling model with the scalar field transfers energy into the matter, in which the rate is taken to be proportional to the matter density, $Q \propto-H \rho_{m}$, i.e., $\Gamma \propto-x$. The dependence upon $\Gamma$ is demonstrated in Fig. 4, and larger values of $\Gamma$ yield an earlier return of deceleration.

Figs. 5 and 6 show the results for the coupling model in which the matter transfers energy into the scalar field with $\Gamma \propto x$. The dependence upon the parameter $\lambda$ is demonstrated in Fig. 6, and larger values of $\lambda$ yield a shorter period of transient acceleration.

In these plots we extrapolate the model to earlier era. Figs, 1, 3, and 5] reveal that, when the models are extrapolated back to the early stages, $\rho_{\phi}(t)$ will be dominant over $\rho_{m}(t)$ and $\rho_{r}(t)$, i.e., the $\phi$ field is dominant over the matter, and has $w=1$ with $\rho_{\phi}(t)=p_{\phi}(t) \propto a^{-6}(t)$, a feature that has been known [27]. The model predicts a scalar field dominated era for a range of redshift $z>z_{a}$, where $z_{a}$ is some value $\in(4 \sim 20)$, depending upon the sign and value of $\Gamma$ and upon the model parameters. This kind of dynamic behavior for the early era deviates drastically from the scenario of the standard cosmology. Therefore, the class of models with exponential potential for a transient acceleration can be pertinent only for rather recent era during the matter domination stage. They cannot account for the early expansion of the Universe with $z>z_{a}$. The standard BigBang cosmology has a scenario that the matter component should be dominant in the past up to the radiation-and-matter equality at a redshift $z \sim 3450$ [31], and the acceleration era starts rather recently around $z \sim 0.5$. The whole class of models, either $\Gamma=0$, or $\Gamma>0$, or $\Gamma<00$, have this difficulty. To be concordant with the Big Bang scenario in this class of models, one would have to choose a smaller initial value of $\rho_{\phi}$, so that $\rho_{\phi}(t)$ is subdominant to $\rho_{m}(t)$ during the radiation or matter dominated era. Just as investigated in Refs. [32, 33], by constructing the so-called quintessence $\phi$ with a double exponential potential $V(\phi)$, one can achieve such a scaling solution followed by an exit into the accelerating expansion. However, as we have just shown, the dynamic evolution of this double exponential quintessence does not automatically ensure a proper future deceleration. If one still wants to achieve a transient acceleration, one has to use the double exponential quintessence for the scaling stage and the exit stage, and probably employ another new field for the return of deceleration, 
as in Refs. [21, 22]. Still, one would have to give a physical motivation for such an artificial connection. It is still premature yet to compare the above models with the observed data of cosmology, such as SN la [2], CMB [34], and BAO [35]. We mention that the fluid dark energy model with a transient acceleration [30] would also face the same problem as the above.

\section{Conclusion}

We have explored the possibility of transient acceleration in a class of scalar dark energy models with exponential potentials in the presence of the matter and radiation components. By the detailed examinations for three cases, the noncoupling with $\Gamma=0$, the coupling with $\Gamma<0$, and the coupling with $\Gamma>0$, we find that this class of models with exponential potential can provide a transient period of acceleration. However, extrapolating back to the earlier era of redshifts $z \gtrsim 5(\Gamma=0)$, or $z \gtrsim 4(\Gamma<0)$, or $z \gtrsim 20(\Gamma>0)$, respectively, the scalar energy $\rho_{\phi}(t)$ will be dominant over the matter and radiation and the scaling of behavior is lost too soon. This would be inconsistent with the standard BigBang cosmology, in which the matter-dominated era extends from $z \sim 3450$ up to $z \sim 0.5$. The coupling between the scalar dark energy and the matter brings only minor modifications to the dynamic expanding behavior. A greater value of $\Gamma$ tends to yield a shorter period of acceleration and an earlier return of deceleration.

Therefore, as they presently stand, this class of models with exponential potentials can be only used at most from more recent past around low redshifts. In order to have a viable model of transient acceleration, one has to either use the exponential scalar potential to construct more sophisticated models with a proper dynamic behavior at high- $z$, or to seek other models.

ACKNOWLEDGMENT: W.P Cui's work has been part of the Undergraduate Research Project of USTC. Y.Zhang's research work has been supported by the CNSF No.10773009, SRFDP, and CAS.

\section{References}

[1] A. G. Riess et al., Astron. J. 116, 1009 (1998); Astron. J. 117, 707 (1999); Astrophys. J. 607, 665 (2004); S.Perlmutter et al., Astrophys.J. 517,565 (1999) ; J.L.Tonry et al., Astrophys.J. 594,1 (2003); R.A.Knop et al., Astrophys.J. 598,102 (2003) ; W.M.Wood-Vasey et al., astro-ph/0701041. 
[2] Amanullah R et al 2010 Astrophys.J. 716 712. For The Supernova Cosmology Project, the numerical data of the full sample are available at http://supernova.Ibl.gov/Union

[3] S. Hanany et al., Astrophys. J. Lett. 545, L5 2000; C.B. Netterfield et al., Astrophys. J. 571, 604 (2002); E. Komatsu et al., Astrophys. J. Suppl. 180, 330 (2009);

[4] M. Colless et al., Mon. Not. R. Astron. Soc. 328, 1039 (2001); M. Tegmark et al., Phys. Rev. D 69, 103501 (2004); S. Cole et al., Mon. Not. R. Astron. Soc. 362, 505 (2005); V. Springel, C.S. Frenk, and S.M.D. White, Nature (London) 440, 1137 (2006).

[5] S. Boughn and R. Chrittenden, Nature (London) 427, 45 (2004); P. Vielva, E. MartinezCGonzalez, and M. Tucci, Mon. Not. R. Astron. Soc. 365, 891 (2006).

[6] C.R. Contaldi, H. Hoekstra, and A. Lewis, Phys. Rev. Lett. 90, 221303 (2003).

[7] Carroll, S. Living Rev. Relativity. 4, 1(2001).

[8] B. Ratra and P.J.E. Peebles, Phys. Rev. D37, 3406 (1988); P.J.E. Peebles and B.Ratra, ApJ 325, L17 (1988); C. Wetterich, Nucl. Phys. B302, 668 (1988); A\&A 301, 321 (1995); R.R. Caldwell, R. Dave and P.J. Steinhardt, Phys. Rev. Lett. 80, 1582 (1998); I. Zlatev, L. Wang and P.J. Steinhardt Phys. Rev. Lett. 82, 896 (1999).

[9] R.R. Caldwell, Phys. Lett. B 545, 23 (2002); S.M. Carroll, M. Hoffman and M. Trodden, Phys. Rev. D68, 023509 (2003); R.R. Caldwell, M. Kamionkowski and N.N. Weinberg, Phys. Rev. Lett. 91, 071301 (2003).

[10] C. Armendariz-Picon, T. Damour and V. Mukhanov, Phys. Lett. B 458, 209 (1999) ; C. Armendariz-Picon, V. Mukhanov and P.J. Steinhardt, Phys. Rev. D63, 103510 (2001); T. Chiba, T. Okabe and M. Yamaguchi, Phys. Rev. D62, 023511 (2000).

[11] T. Padmanabhan, Phys. Rev. D66, 021301 (2002); J.S. Bagla, H.K. Jassal, and T. Padmanabhan, Phys. Rev. D67, 063504 (2003). 
[12] W. Hu, Phys. Rev. D71, 047301 (2005); B. Feng, X.L. Wang and X.M. Zhang, Phys. Lett. B 607, 35 (2005); W. Zhao and Y. Zhang, Phys. Rev. D73, 123509 (2006).

[13] A.Y. Kamenshchik, U. Moschella and V. Pasquier, Phys. Lett. B 511265 (2001); M.C. Bento, O. Bertolami and A.A. Sen, Phys. Rev. D 66043507 (2002); X. Zhang, F.Q. Wu and J. Zhang, JCAP 0601003 (2006).

[14] Y. Zhang, Gen. Rel. Grav.34, 2155 (2002); Gen. Rel. Grav. 35, 689 (2003); Chin. Phys. Lett.20,1899 (2003); Chin. Phys. Lett. 21, 1183 (2004); W. Zhao and Y. Zhang, Phys. Lett. B 640, 69 (2006); Class. Quant. Grav. 23, 3405 (2006). Y. Zhang, T.Y. Xia, and W. Zhao, Class.Quant.Grav. 24, 3309 (2007).

[15] T.Y. Xia and Y. Zhang, Phys. Lett. B 656, 19 (2007).

S. Wang, Y. Zhang, Phys. Lett. B 669, 201 (2008);

S. Wang, Y. Zhang, and T. Y. Xia, J. Cosmol. Astropart. Phys. 10 (2008) 037;

M. L. Tong, Y. Zhang, and T. Y. Xia, Int. J. Mod. Phys. D 18, 797 (2009).

[16] K. Bamba, S. Nojiri, S. Odintsov, Phys.Rev.D77, 123532 (2008)

[17] A. Shafieloo, V. Sahni and A. A. Starobinsky, Phys. Rev. D 80, 101301 (2009).

[18] W. Fischler, A. Kashani-Poor, R. McNees, S. Paban. JHEP 0107,003 (2001)

[19] P. Townsend, M. Wohlfarth, Phys. Rev. Lett. 91 (2003) 061302, hep-th/0303097.

[20] V. Sahni and Y. Shtanov, J. Cosmol. Astropart. Phys. 11 (2003) 014

[21] J. G. Russo, Physics Letters B 600185 (2004).

[22] F. C. Carvalho, J. S. Alcaniz, J. A. S. Lima, and R. Silva, PRL 97, 081301 (2006)

[23] J.S. Alcaniz, IAU 2009 JD9 conference proceedings. MmSAlt, vol.80 . Eds. Paolo Molaro amp; Elisabeth Vangioni. 
[24] C. Wetterich, Nucl. Phys. B252, 3091985.

J. Halliwell, Nucl. Phys. B266, 228 (1986); Phys. Lett. B 185, 341 (1987).

Q. Shafi and C. Wetterich, Phys. Lett. B152, 51 (1985); Nucl. Phys. B289, 787 (1987).

[25] E. Cremmer et al., Phys. Lett. 133B, 61 1983; J. Ellis et al. Phys. Lett. 134B, 429 1984; J. Yokoyama and K. Maeda, Phys. Lett. B 207, 31 (1988).

[26] J. Barrow and S. Cotsakis, Phys. Lett. B 214, 515 (1988). S. Cotsakis, Phys. Rev. D bf47, 1437 (1993).

[27] P. Ferreira and M. Joyce, Phys. Rev. Lett. 79, 474 (1997); Phys.Rev.D 58, 023503 (1998).

[28] B. Ratra and P. Peebles, Phys. Rev. D 37, 3406 (1988).

P. Ferreira and M. Joyce, Phys. Rev. Lett. 79, 474 (1997); Phys.Rev.D 58, 023503 (1998).

C. Wetterich, Astron. Astrophys. 301, 321 (1995).

E. Copeland, A. Liddle, and D. Wands, Ann. N.Y. Acad. Sci.688, 647 (1993).

A. Albrecht and C. Skordis, Phys.Rev.Lett.84, 2075 (2000).

S. Dodelson, M. Kaplinghat, and E. Stewart, Phys.Rev.Lett.85, 5276 (2000).

[29] F.E.M. Costa and J.S. Alcaniz, Phys.Rev.D81 043506, (2010).

[30] J. C. Fabris, B. Fraga, N. Pinto-Neto, W. Zimdahl, arXiv: 0910.3246, astroph.CO.

[31] Spergel D N et al Astrophys. J. 148175 (2003).

[32] A.A. Sen, S. Sethi, Phys.Lett. B532 159 (2002).

[33] T. Barreiro, E. Copeland, N.J. Nunes, Phys.Rev.D61 127301 (2000)

[34] Komatsu E et al 2011 Astrophys.J.Suppl. 19218.

[35] Percival W J et al 2010 Mon.Not.R.Astron.Soc. 401 2148; Percival W J et al 2007 Mon.Not.R.Astron.Soc. 381 1053; Eisenstein D J et al 2005 Astrophys.J. 633560. 


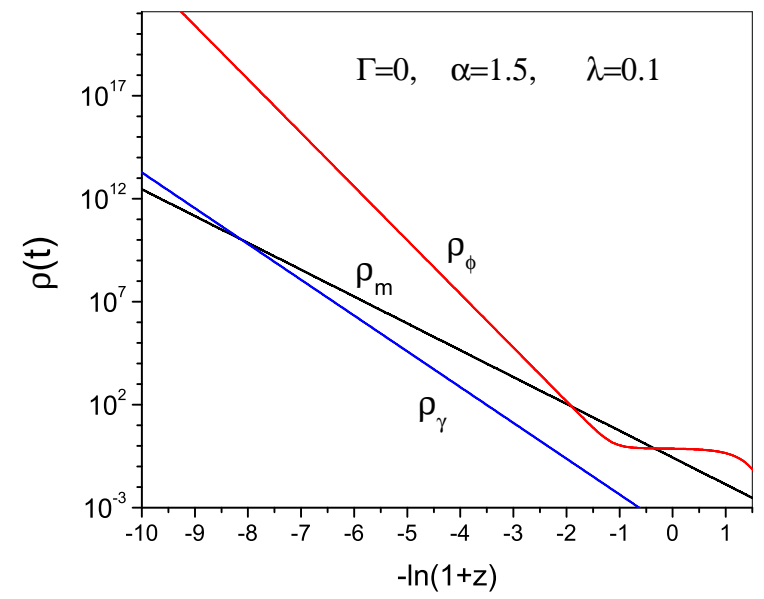

Figure 1: $\rho(t)$ in non-coupling model with $\Gamma=0$. Notice that $\rho_{\phi}$ dominates $\rho_{m}$ and $\rho_{r}$ for $z \gtrsim 5$, which is not compatible with the Big-Bang cosmology.

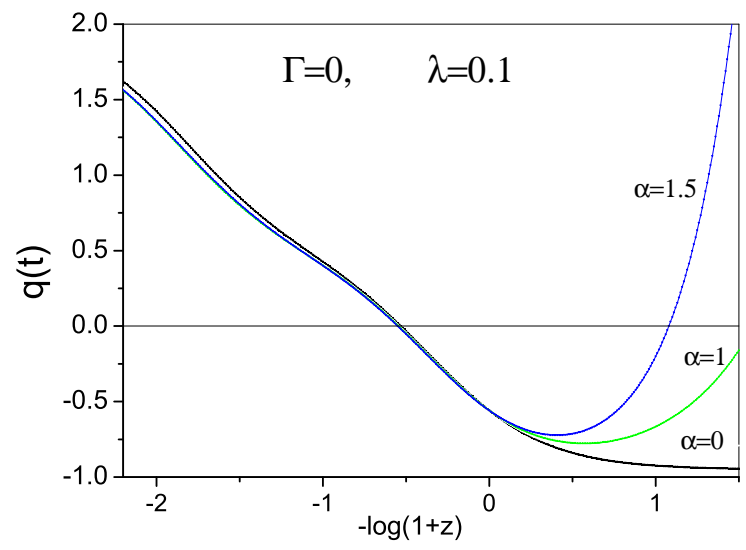

Figure 2: A transient acceleration is shown by the deceleration parameter $q(t)$, which is negative within $a \sim(0.6,2.5)$ for $\alpha=0.15$. A greater $\alpha$ yields a shorter duration of acceleration and an earlier return of deceleration. 


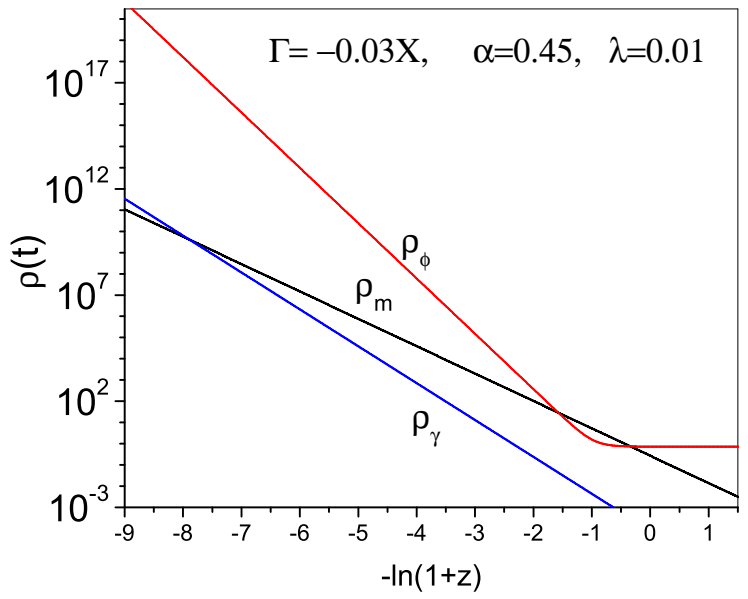

Figure 3: The model $\Gamma<0$. $\rho_{\phi}$ dominates $\rho_{m}$ and $\rho_{r}$ for $z \gtrsim 4$.

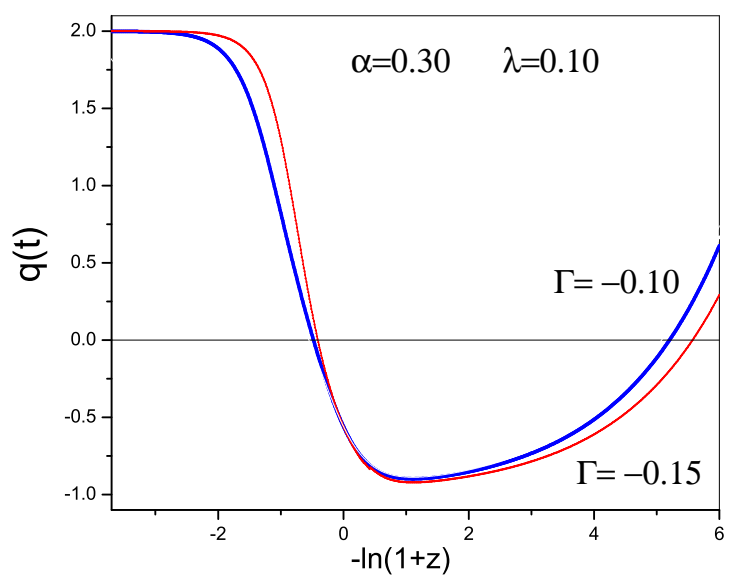

Figure 4: $q(t)$ for various values of $\Gamma<0 . q(t)$ is negative within $a \sim(0.5,5)$. 


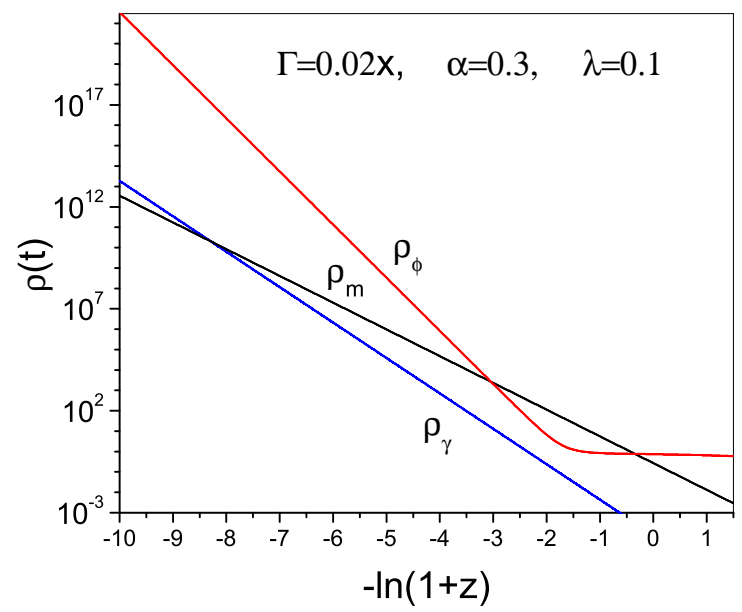

Figure 5: The model $\Gamma>0 . \rho_{\phi}(t)$ dominates $\rho_{m}$ and $\rho_{r}$ for $z \gtrsim 20$.

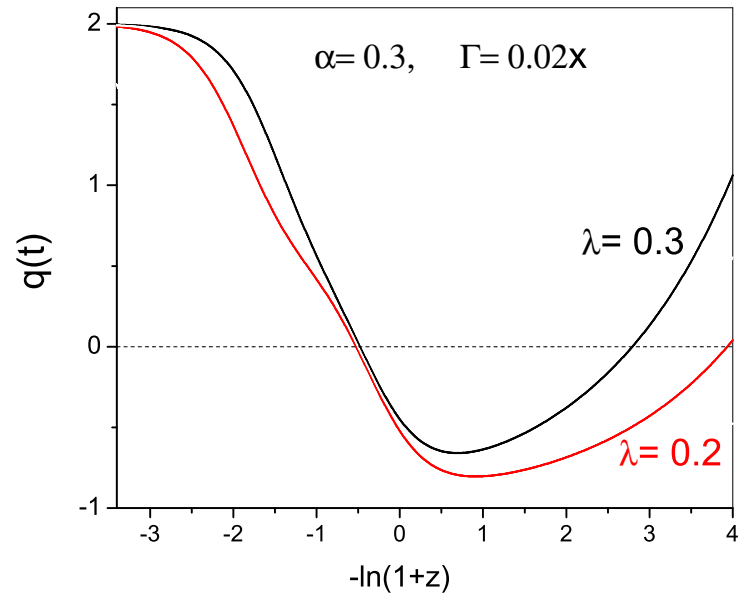

Figure 6: The $\Gamma>0$ model for various $\lambda . q(t)$ is negative within $a \sim(0.5,2.7)$ for $\lambda=0.4$. A greater $\lambda$ yields a shorter duration of acceleration. 